\title{
Using spatial terms to select an object
}

\author{
LAURA A. CARLSON \\ University of Notre Dame, Notre Dame, Indiana \\ and \\ GORDON D. LOGAN \\ Vanderbilt University, Nashville, Tennessee
}

\begin{abstract}
Our interactions with the world often involve selecting one object from a cluttered array of objects. One way to accomplish this is with language. For example, spatial terms, such as above, guide selection by specifying the position of one object (the located object) with respect to a second object (the reference object). Most of the work on the apprehension of spatial terms has examined displays that contain only these two objects. In the present paper, we examine how the presence of an extra object (a distractor) in the display impacts apprehension. Consistent effects of distractor presence were obtained across acceptability-ratingand speeded sentence/picture verification tasks. Importantly, these effects were independent of the placement of the distractor. These results suggest that the distractor has its influence during processes that spatially index and identify the located and reference objects and that processes involved in computing the spatial term operate only on these objects.
\end{abstract}

Our interactions with the world often involve selecting one object from a cluttered array of objects, such as reaching beyond the keyboard for a coffee cup or picking up the stapler that is beside the telephone. In addition to selection by action, we can use language to select objects. For example, we can describe the coffee cup as being behind the keyboard and to the right of the telephone. In this case, successful selection of the coffee cup involves apprehension of the spatial relations that specify its position with respect to nearby objects. More specifically, for the utterance "The coffee cup is behind the keyboard," the object that is intended for selection is the coffee cup (henceforth, the located object), and its location is indicated with reference to the keyboard (henceforth, the reference object). Most of the research in which the apprehension of spatial terms has been examined has focused primarily on the roles played by the reference and the located objects (e.g., Carlson-Radvansky \& Irwin, 1994; CarlsonRadvansky \& Logan, 1997; Clark \& Chase, 1972; Glushko \& Cooper, 1978; Levelt, 1984; Logan, 1995; Logan \& Sadler, 1996; Talmy, 1983; but see Carlson-Radvansky \& Jiang, 1998; Greenspan \& Segal, 1984; Herskovits, 1986; Logan \& Compton, 1996). However, given that the visual world is full of objects, the goal in the present paper was to as-

This work was supported in part by NSF Grants SBR 97-27638 to L.A.C. and SBR 94-10406 and SBR97-09711 to G.D.L. We thank Julie Delheimer for testing the subjects and Robert West for helpful comments. Correspondence concerning this article should be addressed to either L. A. Carlson, Department of Psychology, University of Notre Dame, Notre Dame, IN 46556 (e-mail: lcarlson@nd.edu) or G. D. Logan, Department of Psychology, Vanderbilt University, Nashville, TN 37240 (e-mail: gordon.logan@vanderbilt.edu). sess how the need to select an object from among many objects impacts apprehension. We examined this by systematically manipulating the presence and location of a distractor object in the displays.

\section{THE NEED TO SELECT OBJECTS}

According to Logan and Sadler (1996), the apprehension of spatial terms involves identifying and spatially indexing the reference and located objects, assigning a direction to the spatial term, mapping space around the reference object into regions of acceptability, and evaluating the goodness of fit of the located object with respect to these regions. The direction that is assigned to a spatial term is defined by a reference frame, and the regions of space around the reference object are delineated within a spatial template.

This sequence adequately accounts for apprehension when the reference and the located objects are the only objects present in the display. To assess whether the presence of a distractor would impact the apprehension process, Logan and Compton (1996) presented participants with displays that contained a located object and a reference object embedded in a line of distractors, all vertically arranged. A varying number of distractors ( $0-3)$ was positioned between the located and the reference objects. Sentences of the form "A above B" preceded the displays, and the task was to indicate as quickly and as accurately as possible whether the display contained the letters in the appropriate spatial relation. True trials had the letters in the correct relation; false trials either had the letters present, but in the wrong relation (Experiments 1 and 3), or had the correct relation but were missing one of the letters (Experiment 2). 
Response times were significantly slower when distractors were present than when they were absent. Moreover, response times increased as the number of distractors between the located and the reference objects increased. Logan and Compton (1996) interpreted this effect as reflecting an iterative procedure that involved identifying and indexing the located object. That is, Logan and Compton assumed that subjects started their search for the located object at the reference object, moving in the direction indicated by the spatial term. Each encountered object was then evaluated to determine whether it was the located object. As the number of intervening objects increased, the number of iterations increased.

Thus, distractors seem to influence the processes involved in identifying the objects relevant to the spatial term. The goal of the present paper was to assess whether this influence also operates on processes involved in evaluating the spatial term. According to Logan (1994), spatial terms have compositional meanings (in the sense of Barsalou, 1993; Fodor \& Pylyshyn, 1988) that depend not only on the individual meanings of the located and reference objects, but also on the relation between these objects (see also Garrod \& Sanford, 1989; Herskovits, 1986; Miller \& Johnson-Laird, 1976). That is, not only do the individual objects have to be spatially indexed and identified, but their relation must also be assessed. It is unclear whether the subjects in Logan and Compton's (1996) study were also computing the spatial relation between the distractors and the reference object. Given that the apprehension of spatial terms requires attention (Logan, 1994, 1995), calculation of the spatial relations would be serial. This would predict an increase in response time as a function of the number of intervening distractors, the pattern obtained by Logan and Compton.

If the spatial relationship between the distractor and the reference object is calculated, one might expect the strength of the distractor's influence to vary as a function of whether or not its placement is an acceptable instantiation of the spatial term. For example, Herskovits (1986) has argued that when the placement of a distractor represents a better example of a relation than the placement of the located object, use of the spatial term to describe the located object's placement is prohibited. Such an effect would occur only if the spatial relation between the distractor and the reference object were computed and compared with the relation between the located object and the reference object. For example, for the term above, a distractor that is placed above the reference object should cause more interference than a distractor that is below the reference object. However, Greenspan and Segal (1984) claim that items in the display that are not mentioned in the sentence are not encoded for future use; presumably, their relations with the reference object would not be calculated. According to this view, the influence of the distractor should be restricted to spatially indexing and identifying the located and reference objects, with its strength independent of its placement.

\section{EXPERIMENT 1A}

The goal of Experiment 1A was to examine whether the influence of a distractor varied as a function of its placement. To assess this issue, displays were created in which the distractor, the located object, and the reference object were always vertically aligned but the placement of the distractor was manipulated. Specifically, sometimes the distractor appeared between the located and the reference objects, and sometimes it appeared above or below adjacent located and reference objects. The conditionscorresponding to the term above are depicted in the configuration column in the Distractor Present section of Table 1. Conditions corresponding to the term below are identical, except that the placements of the located and the reference objects were exchanged. The reference object, the located object, and the distractor are represented by the letters R, L, and D, respectively, and the configuration of the objects is represented by the relative positions of the letters. For the distractor-present displays, in the between configuration, the distractor (D) appears between the located object (L) and the reference object (R). In the adjacentcompeting condition, the distractor is above the located object, which, in turn, is above the reference object. The distractor and the located object compete in this condition, in the sense that use of the term above is acceptable as a description of either object's location. In the adjacentnoncompeting condition, the distractor is below the reference object, and the located object is above the reference object.

If the cost obtained in Logan and Compton (1996) was due to an iterative procedure that involved spatially indexing and identifying each object, the influence of the distractor should be constant across these configurations. In all cases, there is one distractor, one located object, and one reference object. However, if the relation between the distractor and the reference object is calculated, the influence of the distractor should differ as a function of how well it instantiates the relation. That is, the distractor should have a more disruptive effect in the adjacentcompeting condition than in the adjacent-noncompeting condition. Indeed, the adjacent-competitor condition may not differ from the between condition, because in both cases, the relation between the distractor and the reference object is the same as the relation between the located object and the reference object (i.e., above).

Distractor-absent conditions were also included. Comparison with the distractor-present conditions enables an assessment of any general influence of the distractor. The conditions for the spatial term above are shown in the configuration column in the Distractor Absent section of Table 1. Conditions for the spatial term below were identical, with the exception that the placements of the located and the reference objects were exchanged. The distractor-absent configurations are labeled as separated, adjacent-bottom, and adjacent-top, respectively, with bottom and top indicating whether the objects occupied the bottom two or the top two positions in the 
Table 1

Configurations and Mean Response Times

(RTs, in Milliseconds) and Accuracy (in Percentages) for Correct True Trials for Experiment 1A and Acceptability Ratings for Experiment 1B

\begin{tabular}{|c|c|c|c|}
\hline \multirow[b]{2}{*}{ Configuration } & \multicolumn{2}{|c|}{ Experiment 1A } & \multirow{2}{*}{$\begin{array}{c}\text { Experiment } 1 \mathrm{~B} \\
\text { Acceptability Rating }\end{array}$} \\
\hline & RT & Accuracy & \\
\hline \multicolumn{4}{|c|}{ Distractor Absent } \\
\hline $\begin{array}{l}\text { Separated } \\
\quad \mathrm{L} \\
- \\
\mathrm{R}\end{array}$ & 859 & 95 & 8.0 \\
\hline $\begin{array}{l}\text { Adjacent-bottom } \\
- \\
\mathrm{L} \\
\mathrm{R}\end{array}$ & 857 & 96 & 8.7 \\
\hline $\begin{array}{l}\text { Adjacent-top } \\
\text { L } \\
\text { R } \\
-\end{array}$ & 860 & 96 & 8.8 \\
\hline \multicolumn{4}{|c|}{ Distractor Present } \\
\hline $\begin{array}{l}\text { Between } \\
\text { L } \\
\text { D } \\
\text { R }\end{array}$ & 1,040 & 93 & 6.5 \\
\hline $\begin{array}{l}\text { Adjacent-competing } \\
\text { D } \\
\text { L } \\
\text { R }\end{array}$ & 1,034 & 92 & 7.9 \\
\hline $\begin{array}{l}\text { Adjacent-noncompeting } \\
\text { L } \\
\text { R } \\
\text { D }\end{array}$ & 1,029 & 95 & 7.9 \\
\hline False trials & 1,008 & 96 & \\
\hline
\end{tabular}

Note-L, located object; R, reference object; D, distractor; - indicates an empty position in the display.

configuration. The "-" in the table indicates an empty position in the configuration; however, it was not actually present in the displays seen by the subjects.

\section{Method}

\section{Subjects}

Thirty-six University of Illinois undergraduates participated and were reimbursed with partial credit or a payment of $\$ 5 / \mathrm{h}$. All gave informed consent and were naive with respect to the hypotheses being tested.

\section{Stimuli and Apparatus}

The stimuli were displayed in white on a dark background on Amdek 722 color monitors controlled by IBM AT computers. Up to 4 subjects could be tested on different computers simultaneously. The stimuli were presented in text mode within the standard IBM $24 \times 80$ text screen. Located objects, distractors, and reference objects appeared in row 11,13 , or 15 , column 38 . On each trial, located objects, reference objects, and distractors were randomly selected without replacement from a set of 20 consonants. Each letter was $5 \mathrm{~mm}$ high and $3 \mathrm{~mm}$ wide, for a visual angle of $0.48^{\circ} \times 0.29^{\circ}$ at a viewing distance of $60 \mathrm{~cm}$. A sentence of the form "Located object ABOVE/BELOW Reference Object" preceded the display, with letters replacing the words "located object" and "reference object." On each trial, the letters and the spatial term were capitalized.

\section{Design}

Each subject performed 576 trials constructed from the following factors: 2 (distractor: presence vs. absence) $\times 3$ (configuration) $\times 2$ (spatial term, above vs. below) $\times 2$ (verity, true vs. false) $\times 24$ (replication). Configuration was nested within distractor presence. The configuration column in Table 1 shows the relevant displays for the term above. When the distractor was present, the configurations were labeled as between, adjacent-competing, and adjacent-noncompeting. When the distractor was absent, the configurations were labeled as separated, adjacent-bottom, and adjacent-top. In terms of verity, for true trials, the letters in the sentence were present in the display in the relation specified in the sentence. The false trials were included to force people to discriminate letter positions and verify the spatial relation. There were three types of false trials: (1) the correct located object and reference objects were present, but in the wrong relation; (2) the located object in the sentence was replaced by a distractor and, hence, was missing from the display; and (3) the reference object in the sentence was replaced by a distractor and, hence, was missing from the display. Each type of false trial was equally represented across the experiment.

\section{Procedure}

The sequence of events on a trial was as follows. A fixation point appeared in the center of the screen (row 13, column 38) for $500 \mathrm{msec}$. It was then replaced by a sentence, with the spatial term centered at the same location as the fixation point. The sentence appeared for $1,000 \mathrm{msec}$ and was then erased. Following a 500-msec pause, the display appeared and remained present until the subjects indicated their judgment. The subjects were asked to determine whether the arrangement of letters in the display matched the statement as quickly and as accurately as possible. They made their responses by pressing either the "Z" key or the "/?" key on the keyboard. The mapping of the keys to responses (e.g., "Z" key to a true response or "Z" key to a false response) was counterbalanced across subjects. Response time was recorded from display onset until response.

\section{Results and Discussion}

Table 1 provides the mean response times and accuracy for correct true trials broken down by configuration and distractor presence. ${ }^{1}$ The mean overall response time and accuracy for correct false trials also appears; however, because these trials could not be analyzed as a function of configuration, we focused on the true trials. For all the analyses in this and all the experiments, a significance level of .05 was adopted, unless otherwise noted.

For response times, there was a significant main effect of distractor, so that distractor-present displays $(M=$ $1,034 \mathrm{msec})$ were verified more slowly than distractorabsent displays $\left[M=859 \mathrm{msec} ; F(1,35)=216.3, M S_{\mathrm{e}}=\right.$ $2,565]$. For percent correct, distractor-present trials were significantly less accurate $(M=94 \%)$ than distractor-absent trials $\left[M=96 \% ; F(1,35)=19.8, M S_{\mathrm{e}}=4.66\right]$. Thus, the presence of the distractor significantly influenced the apprehension of spatial terms. To find out how the distractor had its influence, the distractor-present trials were analyzed separately from the distractor-absent trials.

\section{Distractor-Absent Configurations}

Response times and percent correct were submitted to separate repeated measures analyses of variance (ANOVAs), with configuration (separated, adjacent-top, 
adjacent-bottom) as the critical factor. There was no main effect of configuration for response times or accuracy $(F \mathrm{~S}<1)$. This indicates that the placement of the located object at varying distances from the reference object (compare separated vs. adjacent conditions) did not impact performance, consistent with Logan and Compton's (1996) failure to find distance effects when no distractors were present.

\section{Distractor-Present Configurations}

Response times and percent correct were submitted to separate repeated measures ANOVAs, with configuration (between, adjacent-competing, adjacent-noncompeting) as the critical factor. There was no effect of configuration for response times $(F<1)$ or accuracy $(F<2.3, p>.10)$. This indicates that when the distractor was present, its influence did not depend on its placement. More specifically, whether the distractor was placed in a direction that instantiated the spatial term did not affect performance in a systematic manner (e.g., compare adjacent-competing and adjacent-noncompeting conditions). Thus, there is no evidence that the spatial relation between the distractor and the reference object was being calculated and contrasted with the spatial relation between the located object and the reference object.

\section{EXPERIMENT 1B}

In Experiment 1A, there was a significant effect owing to the presence of a distractor, consistent with previous work (Carlson-Radvansky \& Jiang, 1998; Greenspan \& Segal, 1984; Logan \& Compton, 1996). The significant contribution of Experiment 1A was to demonstrate that this effect was independent of the distractor's placement. This suggests that the locus of the effect is in the processes involved in indexing and identifying the relevant located and reference objects, rather than in those processes involved in computing the spatial term. To obtain converging evidence, in Experiment 1B we tested for an influence of distractor placement in an unspeeded acceptability-rating task. For the apprehension of spatial terms, speeded sentence/picture verification tasks and acceptability-rating judgments have generally shown the same pattern of performance (cf., Carlson-Radvansky \& Irwin, 1993, 1994). However, the decision criteria for the two tasks differ, with the speeded verification judgment biasing dichotomous judgments (i.e., above/not above) and the acceptability-rating task encouraging intermediate judgments. As such, the acceptability-rating task may be more sensitive to distractor placement and may, accordingly, provide a more sensitive test.

\section{Method}

\section{Subjects}

Thirty-six University of Illinois undergraduates participated and were reimbursed with partial credit or a payment of $\$ 5 / \mathrm{h}$. All gave informed consent and were naive with respect to the hypotheses being tested.

\section{Stimuli and Apparatus}

The critical configurations of stimuli are shown in Table 1 . The characteristics of the stimuli and apparatus are the same as those for Experiment 1A.

\section{Design}

Each subject performed 432 trials constructed from the following factors: 2 (distractor: presence vs. absence) $\times 2$ (spatial term: above vs. below) $\times 3$ (configuration) $\times 36$ (replication). All the displays depicted true relations.

\section{Procedure}

The procedure from Experiment 1A was used, with the exception that the subjects were asked to judge how well the conf iguration in the display matched the sentence, using a scale where $1=b a d$ and $9=\operatorname{good}$.

\section{Results and Discussion}

Mean acceptability ratings broken down by distractor presence and configuration are shown in Table 1. There was a main effect of distractor presence, with the configurations for distractor-present trials $(M=7.5)$ considered less acceptable than the configurations for distractorabsent trials $\left[M=8.5 ; F(1,35)=49.9, M S_{\mathrm{e}}=0.40\right]$. Therefore, the presence of the distractor significantly impacted apprehension.

\section{Distractor-Absent Configurations}

Mean acceptability ratings were submitted to a repeated measures ANOVA, with configuration (separated, adjacent-bottom, adjacent-top) as the critical factor. There was a main effect of configuration $[F(2,70)=27.0$, $\left.M S_{\mathrm{e}}=0.22\right]$. Follow-up tests indicated that the separated configuration $(M=8.0)$ was rated as less appropriate than either the adjacent-top configuration $[M=8.8 ; F(1,35)=$ $\left.28, M S_{\mathrm{e}}=0.64\right]$ or the adjacent-bottom configuration $\left[M=8.7 ; F(1,35)=26.2, M S_{\mathrm{e}}=0.65\right]$, with no difference between the last two configurations $(F<1.1)$. This seems to be an effect of distance of the located object from the reference object. However, previous work using acceptabilityrating judgments either has failed to find such an effect (Carlson-Radvansky \& Logan, 1997; Hayward \& Tarr, 1995; Logan \& Compton, 1996; Logan \& Sadler, 1996) or has found a reverse effect, albeit with relatively extreme distances and spatially extended reference objects (Regier \& Carlson, 2001). One difference between the present study and the previous work is that the present experiment used only true displays in which the located object was placed directly above the reference object. In contrast, in the other studies, the located object was placed in many locations around the reference object, including moderately acceptable and bad placements (e.g., Logan \& Sadler, 1996). Therefore, Experiment 1B lacked the contrast set that the other experiments had. This could be important, given that contrast set is known to influence the manner in which objects are described (Eberhard, Spivey-Knowlton, Sedivy, \& Tanenhaus, 1995; Olson, 1970; Tanenhaus, Spivey-Knowlton, Eberhard, \& Sedivy, 1995). Because the only contrast available in Experiment 1B was due to distance, this dimension may have become more salient. 


\section{Distractor-Present Configurations}

Given that the distractor influenced performance, the critical question was whether this impact varied as function of the distractor's placement. Mean acceptability ratings were submitted to a repeated measures ANOVA, with configuration (between, adjacent-competing, adjacentnoncompeting) as the critical factor. There was a main effect of configuration $\left[F(2,70)=49.0, M S_{\mathrm{e}}=0.51\right]$. Followup tests indicated that the between configuration $(M=6.5)$ was rated as less acceptable than either the adjacentcompeting configuration $\left[M=7.9 ; F(1,35)=49.0, M S_{\mathrm{e}}=\right.$ 1.5] or the adjacent-noncompeting configuration $[M=7.9$; $\left.F(1,35)=49.5, M S_{\mathrm{e}}=1.5\right]$. As with the distractor-absent displays, this pattern can be interpreted as an effect of distance. Most important, there was no difference between the two adjacent configurations $(F<1)$. Thus, whether or not the distractor also instantiates the term has no impact on apprehension. This result is consistent with the response time findings from Experiment 1A. Together, Experiments $1 \mathrm{~A}$ and $1 \mathrm{~B}$ indicate that the influence of the distractor is independent of its placement.

\section{EXPERIMENT 2A}

A different test of the idea that the placement of the distractor may influence the apprehension of spatial terms is to systematically manipulate how well both the distractor and the located object instantiate the spatial term. In Experiments 1A and 1B and in Logan and Compton (1996), all of the objects (distractor, reference, and located) were vertically aligned and fell along the axis of the reference frame. Such placements are consistently considered the best examples of a spatial term (Carlson-Radvansky \& Logan, 1997; Hayward \& Tarr, 1995; Logan \& Sadler, 1996). However, it is possible that the distractor's placement may have an influence when the placement of the located object offers a less acceptable example of the term. Consistent with this idea, Herskovits (1986) argues that the acceptable use of a spatial term depends on the context in which the term is used (see also Carlson-Radvansky, Covey, \& Lattanzi, 1999; Coventry, Carmichael, \& Garrod, 1994; Vandeloise, 1991; van der Zee, 1996). For an example based on Herskovits (1986), ${ }^{2}$ the spatial term above is an adequate description of the spatial configuration of the objects A and X in Figure 1A ("A is above X"). However, according to Herskovits, above would not be suitable to describe their configuration in Figure $1 \mathrm{~B}$ because of the presence of object B. In most cases, B, and not A, would be considered above $\mathrm{X}$ (although under some circumstances, one might say that both $\mathrm{A}$ and $\mathrm{B}$ are above $\mathrm{X}$ ). Thus, the applicability of using a given spatial term to describe A's placement with respect to $X$ changes as a function of the presence of $\mathrm{B}$, a distractor. This could take place only if the distractor's relation to the reference object were also being compared and contrasted with the located object's relation to the reference object.

In order to determine whether the meaning of the spatial term changes in the manner that Herskovits (1986) suggests,
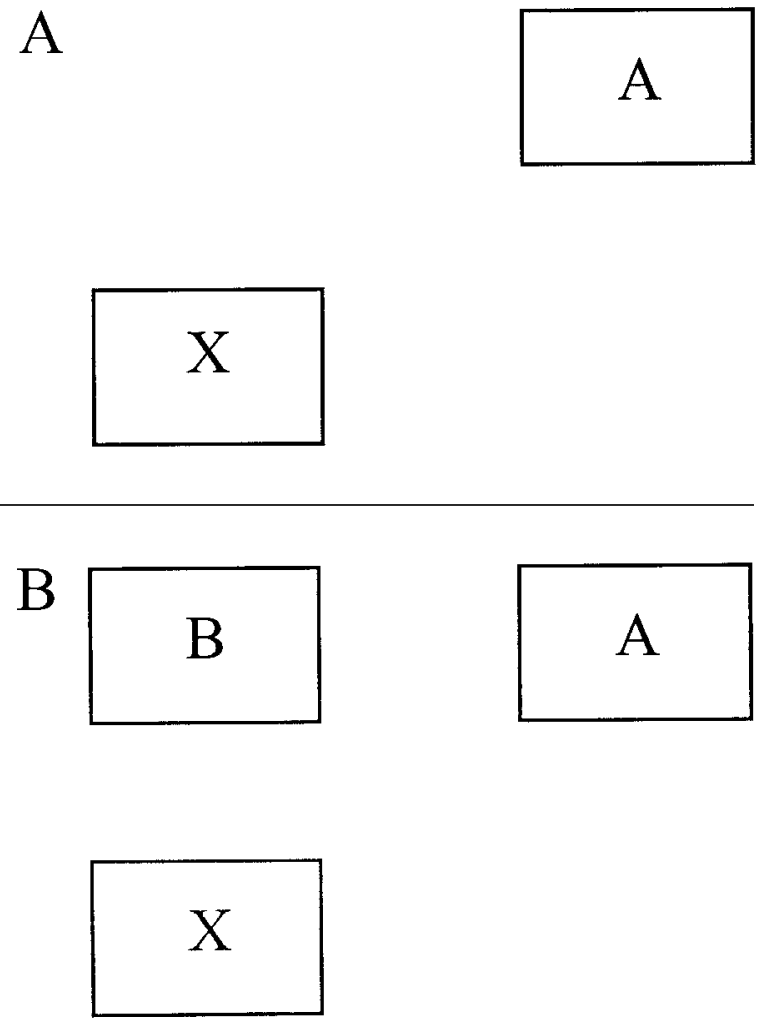

Figure 1. The sentence " $A$ is above $X$ " may be an acceptable description of the configuration in panel $A$, but perhaps not in panel $B$, given the presence of the distractor B. From Language and Spatial Cognition: An Interdisciplinary Study of the Prepositions of English (p. 81), by A. Herskovits, 1986, Cambridge: Cambridge University Press. Copyright 1986 by Cambridge University Press. Adapted with permission.

one needs to manipulate the regions in which the located object and the distractor are placed. Logan and Sadler (1996) divided space around the reference object into three regions. The good region encompasses placements falling along the axis of the reference frame. These placements are generally considered the best examples of a spatial term and are rated as highly acceptable. The acceptable region flanks the good region; these placements are generally rated as moderately acceptable. The $b a d$ region encompasses points in the other direction on the reference frame (i.e., below); these placements are generally rated as not acceptable. In Figure 1B, Object B is in the good region, and $\mathrm{A}$ is in the acceptable region. Thus, because $\mathrm{B}$ is a better example of the spatial term than is A, use of the term to describe A's placement is prohibited.

In Experiment 2, we manipulated the placements of the located object and the distractor across good, acceptable, and bad regions of space. The conditions of interest are shown in the configuration column of Table 2. There were three critical factors: distractor presence, distractor placement, and configuration. The placement factor refers to whether the distractor was placed next to the located object or next to the reference object. The configuration 
factor had three levels, nested within distractor placement. When the distractor was next to the located object, the levels were LG/DA (located object in good region and distractor in acceptable region), LA/DG (located object in acceptable region and distractor in good region), and LA/DA (located object and distractor in acceptable region). (The fourth case [LG/DG] was examined in Experiments 1A and 1B and in Logan \& Compton, 1996.) When the distractor was next to the reference object, the levels were LG/DB (located object in good region and distractor in bad region) and LA/DB (located object in acceptable region and distractor in bad region). ${ }^{3}$ Finally, Table 2 also provides the configurations in which the distractor was absent; on these trials, the located object could be placed in the good or the acceptable region.

The critical question is whether the apprehension process would be affected by the placement of the located object and distractor across different regions of space surrounding the reference object.

\section{Method}

\section{Subjects}

Sixty-eight University of Illinois undergraduates participated in exchange for partial credit or a payment of $\$ 5 / \mathrm{h}$. All gave informed consent and were naive with respect to the hypotheses being tested.

\section{Stimuli and Apparatus}

The critical configurations are shown in the configuration column of Table 2. The characteristics of the stimuli and apparatus are the same as those for Experiment $1 \mathrm{~A}$.

\section{Design}

Each subject performed 576 trials, constructed from the following factors: 2 (distractor, presence vs. absence) $\times 2$ (distractor placement, next to located object vs. next to reference object) $\times 3$ (configuration, nested in placement) $\times 2$ (spatial term, above vs. below) $\times 2$ (verity, true vs. false $) \times 2$ (side) $\times 6$ (replication). In terms of verity, for true trials, the letters in the sentence were present in the display in the relation specified in the sentence. For these trials, the located object was always presented in either the good or the acceptable region around the reference object. False trials were created as in Experiment 1A. Side was a simple counterbalancing factor referring to the side of the display at which the located object appeared.

\section{Procedure}

The procedure was identical to that in Experiment 1A.

\section{Results and Discussion}

Table 2 provides mean response times and accuracy for correct true trials broken down by distractor presence, distractor placement (next to located object vs. next to reference object), and located object placement (good, acceptable, or bad region), collapsing across the side and replication factors. The mean overall response time and accuracy for false trials also appears; however, because these trials could not be analyzed as a function of the critical factors, we focused our analyses on the true trials.

For response times, there was a significant main effect of distractor presence, so that distractor-present displays ( $M=1,102 \mathrm{msec}$ ) were verified more slowly than distractor-absent displays $[M=969 \mathrm{msec} ; F(1,67)=$
Table 2

Configurations and Mean Response Times

(RTs, in Milliseconds) and Accuracy (in Percentages)

for Correct True Trials and for False Trials for Experiment 2A and Acceptability Ratings for Experiment 2B

\begin{tabular}{|c|c|c|c|}
\hline \multirow[b]{2}{*}{ Configuration } & \multicolumn{2}{|c|}{ Experiment $2 \mathrm{~A}$} & \multirow{2}{*}{$\begin{array}{c}\text { Experiment 2B } \\
\text { Acceptability Rating }\end{array}$} \\
\hline & RT & Accuracy & \\
\hline \multicolumn{4}{|c|}{ Distractor Absent } \\
\hline $\begin{array}{r}\mathrm{LG} \\
\mathrm{L} \\
\mathrm{R}\end{array}$ & 958 & 95 & 8.8 \\
\hline $\begin{array}{r}\text { LA } \\
\text { L }\end{array}$ & 975 & 94 & 6.3 \\
\hline
\end{tabular}

Distractor Present

Distractor next to reference object

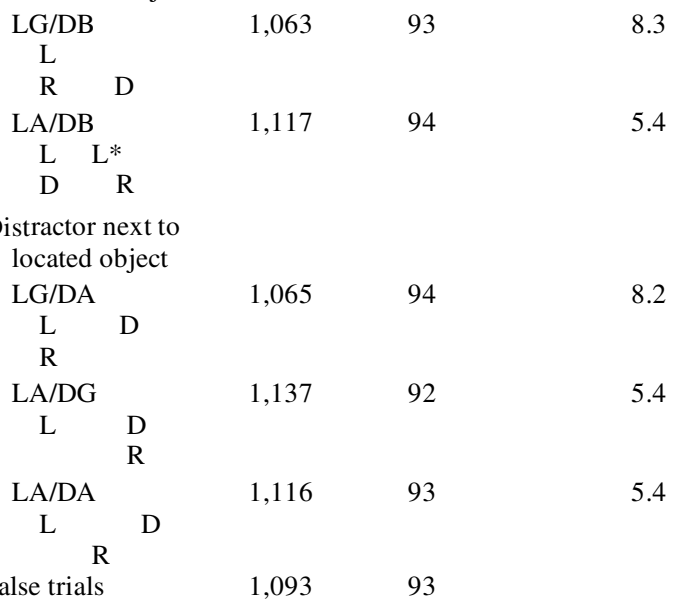

Note-L, located object; $\mathrm{R}$, reference; $\mathrm{D}$, distractor; $\mathrm{G}$, placement in a good region; A, placement in an acceptable region; $\mathrm{B}$, placement in a bad region. *The two Ls shown in the LA/DB configuration indicate two possible placements of the located object. Results are collapsed across these placements.

405.7, $\left.M S_{\mathrm{e}}=1,480.3\right]$. For accuracy, distractor-present trials were less accurate $(M=93 \%)$ than distractor-absent trials $\left[M=94 \% ; F(1,67)=15.2, M S_{\mathrm{e}}=4.4\right]$. Thus, the presences of the distractor significantly influenced the apprehension of spatial terms.

\section{Distractor-Absent Configurations}

Response times and accuracy were submitted to separate repeated measures ANOVAs, with located object placement (good vs. acceptable) as the critical factor. Responses to placements in the good region were significantly faster $(M=958 \mathrm{msec})$ and marginally significantly more accurate $(M=95 \%)$ than responses to placements in the acceptable region $[M=975 \mathrm{msec}$ and $94 \% ; F(1,67)=$ $4.7, M S_{\mathrm{e}}=2,008.0$ and $F(1,67)=3.4, M S_{\mathrm{e}}=12.2, p=.07$, for response times and accuracy, respectively]. Overall, access to the objects placed in the good region was easier than access to objects in the acceptable region, consistent with a trend found by Carlson-Radvansky and Logan (1997, Experiment 2). 


\section{Distractor-Present Configurations}

Because the configurations differed when the distractor was placed next to the located object versus next to the reference object (see Table 2), the results were analyzed separately as a function of distractor placement.

Distractor next to reference object. For these configurations, the located object was placed in the good or the acceptable region, and the distractor was placed in the bad region, thereby failing to instantiate the spatial term. Mean response times and accuracy were submitted to separate repeated measures ANOVAs, with configuration (LG/ DB, LA/DB) as the critical factor, collapsing across the two displays constituting the LA/DB configuration. There was a significant effect of configuration in the response times $\left[F(1,67)=22, M S_{\mathrm{e}}=4,410\right]$, indicating that placements of the located object in the good region $(M=$ $1,063 \mathrm{msec}$ ) were responded to faster than placements of the located object in the acceptable region $(M=$ $1,117 \mathrm{msec})$. There was no effect of configuration in the accuracy data $(F<1.4)$.

Distractor next to located object. For these configurations, both the distractor and the located object were placed in the good or the acceptable region, and thus both instantiated the spatial term. Mean response times and accuracy were submitted to separate repeated measures ANOVAs, with configuration (LG/DA, LA/DG, and LA/ DA) as the critical factor. There was a significant effect of configuration in the response times $[F(2,134)=12.32$, $\left.M S_{\mathrm{e}}=7,502.9\right]$, but not in accuracy $(F<2.0, p>.14)$. Accordingly, follow-up analyses were conducted to determine whether placement of the distractor or the located object influenced response times. First, compare placement of the distractor in a good region (LA/DG, $M=$ $1,137 \mathrm{msec}$ ) with placement of the distractor in an acceptable region (LA/DA, $M=1,116$ ), while holding constant placement of the located object in the acceptable region. These configurations did not significantly differ $\left[F(1,67)=1.8, M S_{\mathrm{e}}=17,102, p>.18\right]$. Counter to the suggestion by Herskovits (1986), performance did not vary depending on whether the distractor instantiated a better example of the spatial term (i.e., LA/DG) than an equally good example (i.e., LA/DA).

Second, compare placement of the located object in the good region (LG/DA, $M=1,065 \mathrm{msec}$ ) with placement of the located object in the acceptable region (LA/DA, $M=$ $1,116 \mathrm{msec}$ ), while holding constant placement of the distractor in the acceptable region. There was a significant difference between these configurations $[F(1,67)=19.7$, $\left.M S_{\mathrm{e}}=8,856.4\right]$, indicating that placement of the located object across the different regions did influence performance. This replicates the effect of easier access to the good region than to the acceptable region obtained in both the distractor-absent configurations and the configurations with the distractor next to the reference object.

\section{Comparisons Across Distractor Placements}

Finally, two critical comparisons across distractor placement (by located object or by reference object) further illustrate that the influence of the distractor is inde- pendent of its actual placement. First, when the located object was placed in the good region, there was no difference owing to placing the distractor in an acceptable region (LG/DA, $M=1,063 \mathrm{msec}$ ) or bad region (LG/DB, $M=1,065 \mathrm{msec} ; t<1)$. Second, when the located object was placed in the acceptable region, there was no difference owing to placing the distractor in the good region (LA/ $\mathrm{DG}, M=1,137 \mathrm{msec}$ ), the acceptable region (LA/DA, $M=$ $1,116 \mathrm{msec}$ ), or the bad region (LA/DB, $M=1,117 \mathrm{msec}$; all $t \mathrm{~s}<1.4)$.

\section{Summary}

There was a consistent and significant influence of the placement of the located object in the good or the acceptable region; however, there was no corresponding influence of distractor placement when it was in the good or the acceptable region.

\section{EXPERIMENT 2B}

In Experiment 2B, an acceptability-rating task was used to obtain converging evidence of an influence of located object placement, but not of distractor placement, across various regions of the spatial template.

\section{Method}

\section{Subjects}

Thirty-six University of Illinois undergraduates participated in exchange for partial credit or a payment of $\$ 5 / \mathrm{h}$. All gave informed consent and were naive with respect to the hypotheses being tested.

\section{Stimuli and Apparatus}

The characteristics of the stimuli and apparatus are the same as those for Experiment 1A.

\section{Design}

Each subject performed 480 trials constructed from the following factors: 2 (distractor, presence vs. absence) $\times 2$ (spatial term, above vs. below) $\times 2$ (distractor placement, by located object vs. by reference object) $\times 3$ (configuration, nested in distractor placement $) \times 2$ (side) $\times 10$ (replication factor).

\section{Procedure}

The procedure was identical to that in Experiment 1B.

\section{Results and Discussion}

Mean acceptability ratings are shown in Table 2, collapsing across side and replication factors. There was a significant main effect of distractor, with distractor-present displays $(M=6.3)$ rated as less acceptable than distractorabsent displays $\left[M=7.1 ; F(1,35)=13.0, M S_{\mathrm{e}}=0.90\right]$. Thus, the presence of the distractor influenced the apprehension process.

\section{Distractor-Absent Configurations}

Mean acceptability ratings were compared across placements of the located object, using a repeated measures ANOVA. Good placements of the located object $(M=8.8)$ received significantly higher ratings than did acceptable placements $\left[M=6.3 ; F(1,35)=245.8, M S_{\mathrm{e}}=0.46\right]$, thus replicating the effects found in both the verification task 
of Experiment 2A and earlier work on spatial templates (e.g., Carlson-Radvansky \& Logan, 1997; Logan \& Sadler, 1996).

\section{Distractor-Present Configurations}

Mean acceptability ratings are presented in Table 2. Separate analyses were conducted as a function of distractor placement.

Distractor next to reference object. For these configurations, the located object was placed in the good or the acceptable region, but the distractor was placed in a bad region, thereby failing to instantiate the spatial term. Mean acceptability ratings were submitted to a repeated measures ANOVA, with configuration (LG/DB, LA/DB) as the critical factor. Placements of the located object in the good region $(M=8.3)$ were rated significantly higher than placements of the located object in the acceptable region $\left[M=5.4 ; F(1,35)=206.7, M S_{\mathrm{e}}=0.71\right]$.

Distractor next to located object. For these configurations, the distractor and the located object were placed in the good or the acceptable region; thus, both instantiated the spatial term. A repeated measures ANOVA, with configuration (LG/DA, LA/DG, and LA/DA) as the critical factor, revealed a significant main effect $[F(2,70)=$ $\left.152.8, M S_{\mathrm{e}}=0.63\right]$. With the placement of the located object in the acceptable region held constant, ratings for placements of the distractor in the good region (i.e., LA/DG, $M=5.4$ ) did not differ from ratings for placements of the distractor in the acceptable region (i.e., LA/DA, $M=5.4 ; F<1)$. This indicates that the placement of the distractor did not impact performance. This finding is also inconsistent with the predictions derived from the Herskovits (1986) example illustrated in Figure 1 , in which the relation between the distractor and the reference object is computed and contrasted with the relation between the located object and the reference object.

In contrast, with the placement of the distractor in the acceptable region held constant, placement of the located object in the good region (LG/DA, $M=8.2$ ) was rated significantly higher than placement of the located object in the acceptable region (LA/DA, $M=5.4$ ). This indicates a significant effect of located object placement, consistent with both the distractor-absent ratings and the response time results of Experiment 2A.

\section{Comparisons Across Distractor Placements}

Finally, two critical comparisons across distractor placement further illustrate that the influence of the distractor is independent of its actual placement. First, when the located object was placed in the good region, there was no difference owing to placing the distractor in an acceptable region (LG/DA, $M=8.2 \mathrm{msec}$ ) or a bad region (LG/DB, $M=8.3$; $t<1.9)$. Second, when the located object was placed in the acceptable region, there was no difference owing to placing the distractor in the good region (LA/DG, $M=5.4$ ), the acceptable region (LA/DA, $M=5.4$ ), or the bad region (LA/DB, $M=5.4$; all $t \mathrm{~s}<1.0$ ).

In summary, consistent with the results from Experiments $1 \mathrm{~A}, 1 \mathrm{~B}$, and $2 \mathrm{~A}$, the influence of the distractor does not depend on its placement in different regions of space around the reference object.

\section{GENERAL DISCUSSION}

Spatial terms assist in the selection of an object by specifying its location with respect to an object whose location is known. Previous research has demonstrated that the presence of a distractor influences the apprehension of spatial terms, with the stage at which the reference and located objects are identified and indexed implicated as the site of this influence. However, whether the distractor affects other processes involved in computing, the spatial relation has not been systematically examined. The goal of the present paper was to determine whether the relationship between the distractor and the reference object is calculated and compared against the relationship between the distractor and the located object.

\section{Distractor Presence}

Across all experiments, there was a consistent and significant effect of distractor presence, such that including a distractor in the display made the verification process more difficult (Experiments 1A and 2A) and reduced acceptability ratings (Experiments $1 \mathrm{~B}$ and $2 \mathrm{~B}$ ). This influence was localized at the stage at which the located and reference objects are spatially indexed and identified. As such, it is perhaps surprising that the distractor significantly reduced acceptability ratings. Acceptability-rating judgments are thought to be an untimed task, so that difficulty at an earlier stage in processing should not ultimately influence performance. One way to interpret this finding is to assume that the rating reflects the ease with which the subjects made their acceptability judgment, consistent with the fluency heuristic in the memory literature (e.g., Jacoby, 1991; Johnston, Dark, \& Jacoby, 1985; Mandler, 1980; Poldrack \& Logan, 1997). Any difficulty that is encountered during the process hinders this ability, and this translates into lower acceptability ratings. If this is true, a difficulty occurring within any of the processes involved in spatial apprehension (e.g., spatial indexing) should translate into lower acceptability ratings.

\section{Distractor Placement}

Most important, when present, the actual placement of the distractor did not influence performance. Counter Herskovits (1986), it did not matter whether the distractor also instantiated the term. For example, ratings and response times did not vary systematically across conditions in which the distractor was placed in accordance with the spatial term (Experiments 1A and 1B). In addition, responses did not vary systematically according to whether the distractor was placed in a good, an acceptable, or a bad region, with the located object in the acceptable region (Experiments 2A and 2B). These findings indicate that the distractor's relation with the reference object is not computed and that the influence of the distractor is on the stages involved in selecting the appropriate located and reference objects. This is consistent with the 
suggestion of previous research (e.g., Carlson-Radvansky \& Jiang, 1998; Logan \& Compton, 1996). For example, Carlson-Radvansky and Jiang dissociated the influence of the distractor on the selection of the relevant objects in the display from selection of an appropriate reference frame. The contribution of the present work is to offer direct evidence in favor of this idea by clearly demonstrating that the influence of the distractor is independent of its placement.

\section{Located Object Placement}

In contrast to the failure to find an effect of distractor placement, there was a significant effect due to placement of the located object across different regions of the spatial template (Experiments 2A and 2B). Specifically, responses were faster and acceptability ratings were higher when the located object was in the good region than when it was in the acceptable region. This effect can be explained by assuming some variability in the assessment of goodness. Greater overlap between the distributions of goodness for located objects in the acceptable and the bad regions than between distributions of goodness for located objects in the good and the bad regions would produce slower response times for located objects in the acceptable region than for located objects in the good region. This effect is noteworthy for a number of reasons. First, it demonstrates that our paradigms are sensitive enough to detect an effect of object placement. Second, together with distractor influence, it suggests an ordering to the processes that underlie the apprehension process. As was outlined in the introduction, apprehension involves the following steps: identifying and spatially indexing the reference and located objects; assigning a direction to the spatial term; mapping space around the reference object into regions of acceptability; and evaluating the goodness of fit of the located object with respect to these regions (see Carlson-Radvansky \& Logan, 1997, and Logan \& Sadler, 1996, for more details). Up to now, no commitment has been made to the independence of these steps or their serial nature. These results permit an initial partitioning and sequencing of the steps involved in spatially indexing and identifying the relevant objects and computing the goodness of fit by demonstrating separable influences at two stages (the overall influence of the distractor that is attributed to the early stage of indexing and identifying, and the effect of located object placement that is attributed to the later stage of computing goodness of fit). Further work should adopt the approach of the present experiments, in which relevant factors (e.g., functional relations) are manipulated systematically across displays to further elucidate the nature of these steps.

\section{REFERENCES}

Barsalou, L. W. (I993). Flexibility, structure, and linguistic vagary in concepts: Manifestations of a compositional system of perceptual symbols. In A. C. Collins, S. E. Gathercole, \& M. A. Conway (Eds.), Theories of memory (pp. 29-101). London: Erlbaum.

Braine, L. G. (1978). A new slant on orientation perspective. American Psychologist, 33, 10-20.
Carlson-Radvansky, L. A., Covey, E. S., \& Lattanzi, K. L. (1999). "What" effects on "where": Functional influences on spatial relations. Psychological Science, 10, 516-521.

Carlson-Radvansky, L. A., \& Irwin, D. E. (1993). Frames of reference in vision and language: Where is above? Cognition, 46, 223-244.

CARlson-Radvansky, L. A., \& IRWIN, D. E. (1994). Reference frame activation during spatial term assignment. Journal of Memory \& Language, 33, 646-671.

Carlson-RadVAnsky, L. A., \& JiAng, Y. (1998). Inhibition accompanies reference frame selection. Psychological Science, 9, 386-391.

Carlson-Radvansky, L. A., \& Logan, G. D. (1997). The influence of reference frame selection on spatial template construction. Journal of Memory \& Language, 37, 411-437.

Clark, H. H., \& Chase, W. (1972). On the process of comparing sentences against pictures. Cognitive Psychology, 3, 472-517.

Coventry, K. R, Carmichael, R., \& Garrod, S. C. (1994). Spatial prepositions, object-specific function, and task requirements. Journal of Semantics, 11, 289-309.

Eberhard, K. M., Spivey-Knowlton, M. J., Sedivy, J. C., \& TanenHAUS, M. K. (1995). Eye movements as a window into real-time spoken language comprehension in natural contexts. Journal of Psycholinguistic Research, 24, 409-436.

Fodor, J. A., \& Pylyshy n, Z W. (1988). Connectionism and cognitive architecture: A critical analysis. Cognition, 28, 3-71.

Garrod, S. C., \& SANFord, A. J. (1989). Discourse models as interfaces between language and the spatial world. Journal of Semantics, $\mathbf{6}$, $147-160$

Glushko, R. J., \& Cooper, L. A. (1978). Spatial comprehension and comparison processes in verification tasks. Cognitive Psychology, 10, 391-421.

Greenspan, S. L., \& Segal, E. M. (1984). Reference and comprehension: A topic-comment analysis of sentence-picture verification. $\mathrm{Cog}$ nitive Psychology, 16, 556-606.

HaYWARD, W. G., \& TARr, M. J. (1995). Spatial language and spatial representation. Cognition, 55, 39-84.

HersKovits, A. (1986). Language and spatial cognition: An interdisciplinary study of the prepositions of English. Cambridge: Cambridge University Press.

JACOBY, L. L. (1991). A process dissociation framework: Separating intentional from automatic uses of memory. Journal of Memory \& Language, 30, 513-541.

Johnston, W. A., DARK, V., \& JACOBY, L. L. (1985). Perceptual fluency and recognition judgments. Journal of Experimental Psychology: Learning, Memory, \& Cognition, 17, 210-233.

LEvelt, W. J. M. (1984). Some perceptual limitations on talking about space. In A. J. van Doorn, W. A. van der Grind, \& J. J. Koenderink (Eds.), Limits in perception (pp. 323-358).Utrecht: VNU Science Press.

LoGAN, G. D. (1994). Spatial attention and the apprehension of spatial relations. Journal of Experimental Psychology: Human Perception \& Performance, 20, 1015-1036.

Logan, G. D. (1995). Linguistic and conceptual control of visual spatial attention. Cognitive Psychology, 28, 103-174.

Logan, G. D., \& Compton, B. (1996). Distance and distraction effects in the apprehension of spatial relations. Journal of Experimental Psychology: Human Perception \& Performance, 22, 159-172.

Logan, G. D., \& SADLER, D. D. (1996). A computational analysis of the apprehension of spatial relations. In P. Bloom, M. A. Peterson, L. Nadel, \& M. Garrett (Eds.), Language and space (pp. 493-529). Cambridge, MA: MIT Press.

MANDLER, G. (1980). Recognizing: The judgment of previous occurrence. Psychological Review, 87, 252-271.

McMullen, P. A, \& Jolicceur, P. (1990). The spatial frame of reference in object naming and discrimination of left-right reflections. Memory \& Cognition, 18, 99-115.

Miller, G. A., \& Johnson-Laird, P. N. (1976). Language and perception. Cambridge, MA: Harvard University Press.

OLson, D. (I970). Language and thought: Aspects of a cognitive theory of semantics. Psychological Review, 77, 257-273.

Poldrack, R. A., \& Logan, G. D. (1997). Fluency and response speed in recognition judgments. Memory \& Cognition, 25, 1-10. 
Regier, T., \& CARlson, L. A. (2001). Grounding spatial language in perception: An empirical and computational investigation. Journal of Experimental Psychology: General, 130, 273-298.

TALmy, L. (1983). How language structures space. In H. L. Pick \& L. P. Acredolo (Eds.), Spatial orientation: Theory, research and application (pp. 225-282). New York: Plenum.

Tanenhaus, M. K., Spivey-Knowlton, M. J., Eberhard, K. M., \& SeDIVY, J. C. (1995). Integration of visual and linguistic information in spoken language comprehension. Science, 268, 1632-1634.

VANDELOISE, C. (1991). Spatial prepositions: A case study from French. Chicago: University of Chicago Press.

VAN DER ZEE, E. (1996). Spatial knowledge and spatial language. Unpublished doctoral dissertation, ISOR/Utrecht University.

\section{NOTES}

1. All analyses were collapsed across spatial term (above vs. below). Analyses including spatial term as a factor generally showed faster and more accurate response times (in Experiments 1A and 2A) and higher acceptability ratings (Experiments $1 \mathrm{~B}$ and $2 \mathrm{~B}$ ) for above relative to below, consistent with previous work (e.g., Clark \& Chase, 1972; McMullen \& Jolicœur, 1990). This difference has been attributed to an interpretation of below as marked with respect to above (e.g., Clark \& Chase, 1972; see also Braine, 1978, for a scanning explanation). More important, spatial term did not interact with the effects of interest; consequently, the presented results were collapsed across this variable.

2. Herskovits (1986) provides the following example, but uses the relation to the right of. We changed this to above in order to relate it more directly to our experiments.

3. The LA/DB condition is averaged across two types of displays that both have the located object placed in the acceptable region and the distractor placed in the bad region. The only difference between the displays is that the distractor either appeared under the located object or farther off to the side. Because this distinction is not meaningful, we averaged across these displays for ease of presentation. Importantly, when considered separately, each of these conditions exhibited the same pattern of results, in comparison with the other conditions. A direct comparison between these two displays revealed slower response times when the distractor was under the located object $(M=1,146 \mathrm{msec}, 93 \%$ correct) than when it was off to the side $[M=1,088 \mathrm{msec}, 93 \%$ correct; $\left.F(1,67)=18, M S_{\mathrm{e}}=12,762\right]$. This could be due to the fact that in the former condition, the distractor and the located object formed a vertically aligned pair and that this pair was selected more often initially as candidate objects, as compared with the latter condition, in which no pair of objects was vertically aligned.

(Manuscript received March 2, 2000; revision accepted for publication May 10, 2001.) 